\title{
JUDEOFOBIA Y MITO CONSPIRATIVO EN LA OBRA DE MIGUEL SERRANO
}

\author{
Gustavo Guzmán Castro \\ Licenciado en Historia \\ Universidad de Chile \\ gu.guzman@gmail.com
}

\section{RESUMEN:}

La judeofobia, conocida tradicionalmente como antisemitismo, es una de las más antiguas formas de exclusión social. Aunque comparte ciertas características con fenómenos afines, como la xenofobia y el racismo, posee un rasgo específico que la diferencia y hace única: su carácter -en palabras de Gustavo D. Perednik- "quimérico". A saber, la fuerte tendencia a emanciparse de la realidad cotidiana, en favor de complejos y elucubrados relatos en los que se atribuye a los judíos una fuerza y una maldad inauditas. Estos relatos, que rozan en la superstición, constituyen el mito de la conspiración judía mundial, cuyo ejemplo más clásico son los Protocolos de los Sabios de Sión. En Chile, el principal discurso antijudío de este tipo se encuentra en la obra del escritor Miguel Serrano, quien pretende "explicar" la historia chilena y sudamericana reciente a partir de sus representaciones conspirativas antijudías. En este artículo nos detendremos en dos breves ejemplos, que nos servirán para esbozar un esquema de cómo funciona el discurso judeófobo de Miguel Serrano, discurso que sirve de soporte ideológico para numerosas agrupaciones radicales en el contexto del neonazismo "virtual" contemporáneo.

PALABRAS CLAVE:

Judeofobia - Antisemitismo - Nazismo - Chile - Miguel Serrano

\section{ABSTRACT:}

The judeophobia -usually known as anti-Semitism- is one of the oldest forms of social exclusion. Although it has some features in common with other similar phenomenons, like xenophobia and racism, it has a specific characteristic that makes it unique: its -in Gustavo Perednik's words- "chimerical" character. That means a strong tendency to become emancipated of reality, opting for complex stories where Jews are ridiculously powerful and evil. These superstitious stories constitute the myth of the Jewish worldwide conspiracy, whose best example is The Protocols of the Elders of Zion. In Chile, the main anti-Jewish discourse of this type is the work of the writer Miguel Serrano, who pretends "to explain" the recent History of Chile and South America from his anti-Jewish representations. In this paper we will see two brief examples. Through them we will outline a scheme of how the Miguel Serrano's judeophobic discourse operate, discourse that works as ideological support for many radical groups in the contemporary "virtual" Neonazism context.

KEY WORDS.

Judeophobia - Anti-Semitism - Nazism - Chile - Miguel Serrano 
La judeofobia ${ }^{1}$ contemporánea proviene principalmente de tres frentes: 1) la extrema derecha, en especial del ala neonazi; 2) el fundamentalismo islámico; 3) grupos de extrema izquierda. Éstos últimos centran sus acusaciones en el "lobby judío", la "responsabilidad sionista en la violencia de Oriente Medio, en general" y considera al sionismo una ideología racista y colonialista equiparable al apartheid sudafricano ${ }^{2}$, negando el derecho del estado de Israel a existir. El fundamentalismo islámico comparte gran parte de estas argumentaciones, en especial lo relativo al conflicto árabe-israelí, a las que suma motivos religiosos ${ }^{3}$. Si bien la importancia de estos "nuevos" actores en el estudio de la judeofobia contemporánea es innegable, creemos que el principal foco de discursividad antijudía -por regularidad y radicalidad- sigue siendo la extrema derecha, en particular las agrupaciones de corte neonazi. $Y$, considerando el rol central que juegan los grupos latinoamericanos en el escenario neonazi mundial ${ }^{4}$, el estudio de su discurso antijudío crece en importancia.

La bibliografía dedicada al estudio de la escena neonazi mundial contemporánea indica que la figura de Miguel Serrano es central ${ }^{5}$. Su influencia sobre numerosas agrupaciones respecto de la "cuestión judía" ha sido enorme y, desde la masificación de internet y su utilización por parte del nazismo contemporáneo ${ }^{6}$, ésta no ha dejado de crecer. De ahí el interés por estudiar judeofobia y mito conspirativo en su obra. Por medio de sus innumerables libros, artículos, entrevistas y de su labor editorial, Serrano ha difundido viejas ficciones y representaciones propias, a la luz de las cuales pretende explicar la historia contemporánea.

Estas representaciones antijudías son apetecidas por neonazis -aunque no sólo por ellos- de todo el mundo, incluidos españoles, alemanes, escandinavos y estadounidenses ${ }^{7}$. Y Serrano ha sabido proveer a su público de copioso material: entre los años 1978 y 2003 Serrano escribió diecinueve libros abiertamente antijudíos, además de prologar y editar en Chile cinco textos fundamentales de la judeofobia moderna, como Los Protocolos de los Sabios de Sión y Mi Lucha.

Para organizar el estudio de este material hemos desarrollado la siguiente clasificación que comprende sus publicaciones entre 1978 y 2003, sin considerar artículos y entrevistas:

I. Obras mayores: aquellas que conforman la llamada Trilogía del Hitlerismo Esotérico: El Cordón Dorado. Hitlerismo Esotérico (1978); Adolf Hitler, el Último Avatara (1984); Manú. Por el Hombre que Vendrá (1991). Son las obras judeófobas más importantes de Serrano. Juntas suman más de 1.200 páginas. Han sido publicadas en inglés y alemán.

II. Obras medias: textos más breves y específicos que los anteriores, pero igualmente valiosos para el estudio de su discurso antijudío. En su mayoría fueron escrito en la década del ochenta.

\footnotetext{
${ }^{1}$ Preferimos el término judeofobia a antisemtismo, por dos razones. En primer lugar porque es más preciso: es el odio (más que temor) a los judíos. Según Gustavo Perednik la palabra judeofobia es más precisa, porque en el prefijo señala el verdadero destinatario de esta aversión, el judío, y en el sufijo alude a su carácter irracional. Perednik, 2000, p. 20. En segundo lugar, porque anula contrargumentaciones como las utilizadas por algunos grupos fundamentalistas islámicos que niegan su evidente antisemitismo porque "también somos semitas".

${ }^{2}$ The Project, 1992, p. 8.

${ }^{3}$ The Project, pp. 8-12.

${ }^{4}$ El panorama neonazi latinoamericano, conformado a partir de movimientos y medios, que reivindican en forma explícita la ideología del nacionalsocialismo y el liderazgo representado por Hitler, no constituye un hecho netamente marginal del neonazismo mundial. No se puede hablar, en estos casos, de la existencia de un centro -con sede en Europa y Norteaméricay una periferia -conformada por América Latina-. Por el contrario, el neonazismo latinoamericano forma parte integral del escenario neonazi mundial, lo que queda ejemplificado con la importancia mundial que tienen los servidores de Internet como "Libre Opinión" y el reconocimiento de ideólogos como Miguel Serrano. Caro, 2007, pp. 190-191.

${ }^{5}$ Casals, 2003, p. 189.

${ }^{6}$ Casals, 2003, p. 199.

${ }^{7}$ Goodrick-Clarke, 1998, pp. 221-222.
} 
III. Obras menores: textos muy breves dedicados a temas puntuales. Algunos son panfletos que transcriben discursos de Serrano. La mayoría fueron escritas en la década del noventa.

IV. Obra autobiográfica: escrita entre 1996 y 1999, relaciona la historia personal del autor con su relato "hitlerista". Aborda aspectos de la historia chilena reciente, tales como el plebiscito de 1988 y la detención de Pinochet en Londres.

V. Obras editadas y prologadas por Serrano: cinco "clásicos" de la judeofobia moderna, promovidos por el autor en Chile y otros países de habla hispana.

En esta oportunidad centraremos nuestra atención en las obras de este último grupo. Específicamente en dos de ellas: los Protocolos de los Sabios de Sión y el Plan Andinia. Ellas serían publicadas, respectivamente, bajo los nombres de Los Protocolos de los Sabios de Sión y su aplicación en Chile y El Plan Andinia. Estrategia Sionista para apoderarse de la Patagonia Chilena y Argentina. Por cierto, no nos detendremos a analizar estos libelos en sí, sino los prólogos y comentarios que Serrano preparó para ambos. Creemos que la importancia de estos textos es doble, pues son fruto tanto de su rol de escritor como de activista nazi.

\section{Los Protocolos de los Sabios de Sión y su aplicación en Chile}

A mediados de 1988, "al cumplirse un año del asesinato ritual del héroe Rudolf Hess", Serrano publica en Chile el más famoso de los textos antijudíos: los Protocolos de los Sabios de Sión. Este "clásico" de la judeofobia -que no es sino una falsificación llevada a cabo por la policía secreta zarista a comienzos del siglo XX-constituye la perfecta versión escrita del mito de la conspiración judía mundial, según la cual "existe un gobierno secreto judío que, mediante una red mundial de organismos y organizaciones camuflados, controla partidos políticos y gobiernos, la prensa y la opinión pública, los bancos y la marcha de la economía. Se dice que el gobierno secreto hace todo eso conforme a un plan secular y con el único objetivo de lograr que los judíos dominen el mundo entero, y también se dice que se está acercando peligrosamente al logro de ese objetivo"'. Los Protocolos se basan en la creencia en esta "superstición política""10 según la cual existiría un gobierno secreto mundial en manos judías que controla y dirige la Historia desde las sombras para beneficio propio.

Para darle un perfil más local y facilitar su difusión entre el público chileno, Serrano escribe un breve prólogo que dará su nombre definitivo al libro: Los Protocolos de los Sabios de Sión y su aplicación en Chile. Éste se resume así: desde hace miles de años existe una conspiración secreta judía (ignorada incluso por muchos judíos, que también son sus víctimas ${ }^{11}$ ), que se propone dominar y destruir a los no judíos. Para lograr dicha meta, los judíos se han servido de su más despiadado invento: la usura, que les permite vivir sin trabajar, enriqueciéndose a costa de los gentiles ${ }^{12}$.

El espíritu de esta conspiración es reconocible ya en la Torah y el Talmud ${ }^{13}$. Sin embargo, al entrar en los tiempos modernos, los Sabios de Sión-cerebros de la conspiración- deciden reunirse con el fin de fijar nuevas estrategias, acordes a la época, que les permitan hacerse del poder total del mundo. Deciden convocar a un cónclave en Basilea el año 1897. Es el primer congreso sionista mundial. Y el texto que conocemos como los Protocolos de los Sabios de Sión son las conclusiones de dicho

\footnotetext{
${ }^{8}$ Los Protocolos 1988, p. 1.

${ }^{9}$ Cohn, 1983, p. 19.

${ }^{10}$ Cohn, 1983 p. 21.

${ }^{11}$ Los Protocolos 1988, p. 32.

${ }_{13}^{12}$ Los Protocolos 1988 p. 33.

13 Los Protocolos 1988 p. 32.
} 
congreso ${ }^{14}$, las cuales se habrían filtrado de una manera poco clara. Una vez producida la "revelación" de Los Protocolos al mundo no judío, la conspiración mundial, el gobierno secreto, mueve todos sus hilos para desprestigiarlos ${ }^{15}$, condenándolos a ser ignorados por la masa. Los Protocolos serían, así, un documento revelatorio: los judíos dominan el capitalismo y el comunismo, la masonería y el Vaticano, a todas las sectas religiosas menores, al terrorismo, etc. Así por ejemplo, manipulan el capitalismo y el comunismo para que parezcan contrarios entre sí, despistando a los gentiles, haciéndolos presas más fáciles de sus planes de dominación ${ }^{16}$. Dicho de otro modo, el capitalismo y el comunismo, sólo aparentemente opuestos, son una misma cosa: instrumentos judíos de dominación mundial. Lo mismo ocurriría con la Iglesia católica y la masonería. Según Serrano, la historia contemporánea toda, pero en particular la historia reciente de América del Sur y de Chile, se reducen a la mera aplicación de Los Protocolos ${ }^{17}$. El prólogo concluye exhortando a los "jóvenes camaradas" a dar a conocer este "libro revelador"18.

La edición, hecha en colaboración con el colectivo neonazi español CEDADE a través de su editorial Wotan, fue dedicada a la memoria de Rudolf Hess ${ }^{19}$. Sin embargo, éste no sería el único homenaje que recibió el jerarca nazi de parte de Serrano y los nazis chilenos. Un año antes de la publicación de Los Protocolos de los Sabios de Sión y su aplicación en Chile Serrano había encabezado una ceremonia en honor al recién fallecido Hess.

\section{El Plan Andinia}

La intención original de Serrano tras la muerte de Hess -ocurrida el 17 de agosto de 1987- fue organizar un acto masivo en la Fuente Alemana del Parque Forestal de Santiago. Ante la negativa de las autoridades del régimen a autorizar el evento, Serrano aprovechó la cercanía del cuadragésimo noveno aniversario de la Matanza del Seguro Obrero para reunir a los nazis santiaguinos en el Cementerio General y rendir allí, junto al monolito de los caídos el 5 de septiembre de 1938, homenaje al lugarteniente de Hitler ${ }^{20}$. La ceremonia -en la que se tomó juramento de jóvenes nazis, se entonaron himnos nazis y donde también leyó un discurso el académico Erwin Robertson ${ }^{21}-$ fue ampliamente cubierta por la prensa escrita santiaguina. Y, en lo que nos compete, cabe destacar que tras el final de la ceremonia, marcado por el discurso de Serrano, jóvenes seguidores del escritor repartieron entre los asistentes copias del recién editado El Plan Andinia. Estrategia sionista para apoderarse de la Patagonia Argentina y Chilena ${ }^{22}$.

El origen de esta versión sudamericana de los Protocolos de los Sabios de Sión es argentino. Según Leonardo Senkman, durante los últimos años del justicialismo en el poder, "en la doctrina peronista se cristalizaron ciertos elementos ideológicos (...) para promover un clima de perturbación antisemita. El más importante de estos elementos fue el de la teoría conspirativa de la Sionarquía Internacionar ${ }^{23}$. Este escenario, propicio para interpretaciones conspirativas, alcanzó su clímax con la aparición de El

\footnotetext{
${ }^{14}$ Los Protocolos 1988 pp. 32-33.

${ }^{15}$ Los Protocolos 1988 p. 30.

${ }^{16}$ Los Protocolos 1988., p. 34.

${ }^{17}$ Los Protocolos 1988 p. 35.

${ }^{18}$ Los Protocolos 1988 p. 38.

${ }^{19}$ Los Protocolos 1988 p. 1.

${ }^{20}$ Las Últimas Noticias, 11 de septiembre de 1987, p.8.

${ }^{21}$ Las Últimas Noticias, 6 de septiembre de 1987, p.17.

${ }_{22}^{22}$ La Época, 6 de septiembre de 1987, p. 16.

23 Senkman, 1986, p. 108.
} 
Plan Andinia, texto judeófobo según el cual "el sionismo se propondría segregar parte del territorio de la Patagonia y fundar un Estado judío". El Plan fue denunciado en 1971 por el nacionalista Walter Beveraggi Allende $^{24}$. El parentesco de este texto argentino con los Protocolos de los Sabios de Sión es evidente.

En cuanto a la edición chilena, ésta es presentada como fruto de la colaboración de "nacionalistas chilenos y argentinos" conscientes de que El Plan Andinia afecta a ambos paíse ${ }^{25}$, y está dedicada a "las juventudes nacionalistas de América del Sur, a los hombres libres, a los civiles y militares patriotas, aún no instrumentalizados por la masonería y las iglesias al servicio del Imperialismo de Sión ${ }^{\prime 26}$.

Además de editarlo, Serrano prologa y comenta el libro.

El prólogo se resume así: existe un gobierno mundial secreto en manos judías, que dirige el capitalismo, el comunismo, la masonería, la Iglesia, sectas menores, terrorismo, etc. Este gobierno secreto judío se ha propuesto arrebatar la Patagonia a Chile y a Argentina para construir allí un segundo estado judío ${ }^{27}$. La historia contemporánea de Latinoamérica se hace más clara a la luz de las revelaciones de El Plan Andinia ${ }^{28}$, el cual provee una suerte de clave para la comprensión de dicha historia. Toda la historia reciente del continente está dirigida al cumplimiento del Plan. Así, por ejemplo, Serrano afirma que el conflicto chileno-argentino de fines de la década de 1970 que casi desemboca en guerra sólo es comprensible a la luz de las revelaciones de El Plan: la existencia de regímenes militares en ambos países ponía en jaque el cumplimiento de El Plan, por lo que los conspiradores llevaron a estos países hermanos al borde de la guerra. También "detrás del atentado de muerte contra Pinochet es visible la mano judía ${ }^{29,}$. Y ante el peligro que este plan (que no sólo es político sino también mítico y de sangre) significa para Chile, se hace urgente llevar a cabo una colonización del extremo sur por jóvenes nacionalistas chilenos o nórdicos que frenen el avance sionista en la zona ${ }^{30}$. Finalmente, hace un llamado a los nacionalistas chilenos y argentinos a trabajar unidos $^{31}$.

Luego, en un apartado de comentarios a Los Protocolos incluido en este libro, Serrano afirma que todas las instituciones públicas chilenas están en manos de los judíos o de sus agentes, los masones ${ }^{32}$. Critica a las FF. AA. por su perjudicial desconocimiento del "real fin del judaísmo internaciona ${ }^{\prime 33}$, y porque se han dejado infiltrar por el sionismo, a través de Fernando Matthei, que promueve el neoliberalismo o "supercapitalismo" de Milton Friedman y los "Chicago Boys" ${ }^{34}$ en Chile. Por último, quisiéramos mencionar que ocho días después del acto en el Cementerio General, el crítico literario de Las Últimas Noticias, Luis Sánchez Latorre, alias Filebo, dedicó un espacio de su columna dominical para comentar la publicación de El Plan Andinia ${ }^{35}$. Extrañamente, y pese a

\footnotetext{
${ }^{24}$ Senkman, 1986, p. 110.

${ }^{25}$ El Plan Andinia, 1987 , p. 9

${ }^{26}$ El Plan Andinia p. 5.

${ }^{27}$ El Plan Andinia p. 9.

28 lbíd.

${ }^{29}$ El Plan Andinia p. 10.

${ }^{30}$ El Plan Andinia p. 11.

${ }^{31}$ El Plan Andinia p. 11-12.

${ }^{32}$ El Plan Andinia p. 37.

${ }^{33}$ El Plan Andinia p. 40.

${ }^{34}$ El Plan Andinia p. 55.

${ }^{35}$ Las Últimas Noticias, 13 de septiembre de 1987, p. 30.
} 
reconocer en otra ocasión que es amigo personal de Serrano ${ }^{36}$, su comentario a El Plan Andinia no menciona la obvia participación de éste en la edición.

Judeofobia y mito conspirativo: conclusiones tentativas

La judeofobia, conocida tradicionalmente como antisemitismo, es una de las formas de exclusión social más antigua y difundida que existe. Si bien tiene características en común con otros fenómenos afines tales como la xenofobia y el racismo, la judeofobia es un fenómeno específico, único. Tiene algo que aquéllos no: un carácter "quimérico" ${ }^{\text {"37 }}$. Con esto nos referimos a la tendencia de estos discursos, en especial los surgidos de la cultura neonazi, a emanciparse la realidad perceptible con los sentidos, en favor de intrincados relatos ficticios que ubican a la figura de el judío como el promotor y beneficiario de los más indecibles males de la humanidad, como la mano oculta que desde las sombras maneja la Historia para $\mathrm{si}^{38}$.

Creemos que en el caso de los textos que hemos revisado esto es evidente, y que desde un punto de vista semiótico es posible esbozar algunas conclusiones sobre el modo de funcionamiento del discurso judeófobo-conspirativo de Miguel Serrano:

1. Serrano, como narrador, es dueño de la designación correcta. En su mundo, compuesto por signos de distinto tipo, el contenido está dado de antemano, y sólo basta conocer el orden, el lenguaje (o sea, la correlación entre los elementos de la expresión y los del contenido ${ }^{39}$ ) para acceder a la Verdad. Y él lo conoce. Él sabe qué es qué.

2. Serrano se siente representante de una cultura de signo positivo (conjunto informacional, valórico, etc. desde el cual escribe). Esta cultura correcta, se contrapone a -y en este caso se supone amenazada por- una anticultura de signo negativo ${ }^{40}$. Esa anticultura es judía, y conspira desde hace milenios para destruir a la cultura de signo positivo.

3. Serrano (y también el auditorio al que escribe) tiene cierta "debilidad" por el Libro ${ }^{41}$, por la revelación (en este caso los Protocolos y el Plan Andinia) que sólo unos pocos (ellos) son capaces de interpretar correctamente.

4. La cultura de signo positivo desde la cual escribe el autor, no explicita normas, reglas, valores, conductas, etc. demasiado claras, unívocas. Sin embargo, no quedan dudas de cuáles son las de signo negativo y quién las encarna y promueve entre la humanidad en beneficio propio.

5. Su discurso más que funcionar rígidamente según un corpus de normas relativamente estables, como una gramática, lo hace como un repertorio de empleos ${ }^{42}$, que siempre se puede acomodar con fines antijudíos. Así, hasta los rivales ideológicos más enconados (como el capitalismo y el comunismo) pueden ser reducidos a agentes de un mismo amo, judío.

\footnotetext{
${ }^{36}$ Las Últimas Noticias, 11 de septiembre de 1987, p.8.

${ }^{37}$ Perednik, 2001, p. 31.

38 "El caso de la judeofobia difiere de la xenofobia. No hay que confrontarse con una interpretación incorrecta, porque no se habla de la realidad cotidiana sino de mitos. Se reivindica el odio a los judíos atribuyéndoles comer a no-judíos en el pasado, dominar el mundo en el presente, matar a Dios, inventar el Holocausto, promover las guerras, la esclavitud, el mal. No es fácil contender con argumentos de esta índole. Éste bien puede considerarse el rasgo esencial de la judeofobia. (...) La judeofobia no guarda relación con el mundo actual, y reposa sobre una ficción alimentada por otras ficciones." En Perednik, 2001, pp. 3132. Un razonamiento muy similar aparece en Taguieff, 2003, p. 42.

${ }^{39}$ Lotman, I.; Uspenski, B. 2000, p. 177.

${ }^{40}$ Lotman, I.; Uspenski, B. 2000, p. 178.

${ }^{41}$ Lotman, I.; Uspenski, B. 2000, p. 178.

${ }^{42}$ Lotman, I.; Uspenski, B. 2000, p. 179.
} 
6. La oposición cultural fundamental no será entre principios "ordenado - no ordenado" (y que por ende deben ser ordenado por nosotros), sino entre el binomio "correcto - incorrecto" (no "no correcto"), incluso "verdadero - falso" ${ }^{43}$.

7. La anticultura, percibida como cultura de signo negativo, se expande abusivamente. Todas las culturas "incorrectas" (a ojos de Serrano: capitalismo, comunismo, cristianismo, masonería, etc.) se constituyen en un único sistema ("todos son instrumentos judíos").

8. Aquí, la cultura se ha cerrado sobre sí, ha preferido el esoterismo por sobre cualquier forma de proselitismo. La cultura, de signo positivo, no pretenderá jamás expandirse en el campo de la anticultura, incorrecta. Su propagación allí sólo sería posible por medio de la victoria de la verdad sobre la mentira ${ }^{44}$, aplastando al universo de signo negativo.

9. Por todo esto, el discurso judeófobo de Miguel Serrano, basado en el mito conspirativo, funciona como un sistema cultural orientado predominantemente hacia la expresión ${ }^{45}$.

Esto último implica que el discurso judeófobo del autor funciona como una suerte de programa, que, con cierta flexibilidad, incorpora información a su repertorio y es capaz de adaptar prácticamente cualquier realidad circundante (la obvia rivalidad capitalista-comunista, por ejemplo) a su modo de funcionamiento, y expresarlo en sus propios términos, antijudíos. El mito conspirativo, en especial en su versión judeófoba, sirve para explicarlo todo ${ }^{46}$. La realidad se adapta al lenguaje judeófobo, y luego se interpreta acorde a él, acorde al rumor sobre los judíos.

\footnotetext{
${ }^{43}$ Lotman, I.; Uspenski, B. 2000, p. 180.

${ }^{44}$ Lotman, I.; Uspenski, B. 2000, pp. 183-184.

${ }^{45}$ Lotman, I.; Uspenski, B. 2000, p. 176.

46 "La teoría de la maquinación es una teoría que vale para todo, pues permite denunciar a unos enemigos invisibles, enmascarados, escondidos, mentirosos, e imputarles acciones inconfesables, con tal de que sean acciones criminales. Se difunde una visión del mundo maniquea, una visión fundada en un principio simplista (y paranoico) para la lectura de todos los acontecimientos, principio que se erige en nueva clase de la historia: la causalidad diabólica del "sionismo internacional" o "mundial" que se manifiesta en todas partes, y que, por consiguiente, todo lo explica". Taguieff, 2003, p. 42.
} 


\section{REFERENCIAS BIBLIOGRÁFICAS.}

\section{Fuentes primarias:}

El Plan Andinia. Estrategia Sionista para apoderarse de la Patagonia Chilena y Argentina. Santiago, Alfabeta Impresores, 1987.

Los Protocolos de los Sabios de Sión y su aplicación en Chile. León, Wotan, 1988.

\section{Fuentes secundarias:}

CARO, I. Extremismos de derecha y movimientos neonazis. Berlín, Madrid, Santiago. Santiago de Chile, LOM, 2007.

CASALS, X. Ultrapatriotas. Extrema derecha y nacionalismo de la guerra fría a la era de la globalización. Barcelona, Crítica, 2003.

COHN, N. El mito de la conspiración judía mundial: los Protocolos de los Sabios de Sión. Madrid, Alianza, 1983.

GOODRICK-CLARKE, N. Hitler's Priestess. Savitri Devi, the Hindu-Aryan Myth and Neonazism. New York, New York University Press, 1998.

LOTMAN, I.; USPENSKI, B. "Sobre el mecanismo semiótico de la cultura", en La Semiósfera, III (Semiótica de las Artes y de la Cultura). Madrid, Cátedra, 2

PEREDNIK, G. La judeofobia. Barcelona, Flor del Viento, 2001.

SENKMAN, L. El antisemitismo en la Argentina. Bs. As., Centro Editor de América Latina, 1986.

TAGUIEFF, P.-A. La nueva judeofobia. Barcelona, Gedisa, 2003.

The Project for the Study of the Antisemitism, Anti-jewish Propaganda 1991. Tel Aviv University, 1992. 